Número especial “Convenção internacional sobre meio ambiente e desenvolvimento para integração e cooperação para a sustentabilidade” - Havana/Cuba.

Vol. 25, n. 1, 2020.

Artigo recebido em: 03/02/2020

Artigo aprovado em: 04/03/2020

\title{
LA PARTICIPACIÓN SOCIAL EN PROYECTOS DE INNOVACIÓN AGROPECUARIA LOCAL
}

\section{A PARTICIPAÇÃO SOCIAL EM PROJETOS DE INOVAÇÃO AGRÍCOLA LOCAL}

\section{SOCIAL PARTICIPATION IN LOCAL AGRICULTURAL INNOVATION PROJECTS}

\author{
Mileidi León Miranda. Cuba'. \\ Luis Antonio Barranco Olivera. Cuba². \\ Anabel Díaz Hurtado. Cuba ${ }^{3}$.
}

\section{RESUMEN}

El presente trabajo propone fundamentar la participación social en procesos productivos a partir de proyectos de innovación agropecuaria local. Temáticas que inciden en el desarrollo social de las comunidades rurales. Se pretende sistematizar resultados obtenidos en el proyecto durante sus tres primeras etapas de experiencias, desde la perspectiva sociológica de prácticas participativas asociadas a los proyectos de innovación agropecuaria. El vínculo que se establece entre estas categorías, propicia escenarios favorables para la adquisición de conocimiento por parte de las comunidades rurales. Así se garantiza la protección del ambiente, alimentos sanos, agricultura ecológica y actores sociales conscientes de su papel en estos resultados.

Palabras claves: participación social, innovación agropecuaria, ambiente.

\footnotetext{
${ }^{1}$ mileidi@cesam.vcl.cu. Centro de Estudios y Servicios Ambientales de Villa Clara (CESAMVC).

2 luisbo@uclv.edu.cu. Universidad Central "Marta Abreu" de Las Villas. Cuba.

3 anabeld@uvlc.edu.cu. Universidad Central "Marta Abreu" de Las Villas. Cuba
} 


\title{
RESUMO
}

Este artigo propõe basear a participação social em processos produtivos baseados em projetos de inovação agrícola local. Temas que afetam o desenvolvimento social das comunidades rurais. Pretende-se sistematizar os resultados obtidos no projeto nas três primeiras etapas da experiência, na perspectiva sociológica das práticas participativas associadas aos projetos de inovação agrícola. O vínculo estabelecido entre essas categorias favorece cenários favoráveis à aquisição de conhecimento pelas comunidades rurais. Isso garante a proteção do meio ambiente, alimentação saudável, agricultura orgânica e atores sociais conscientes de seu papel nesses resultados.

Palavras-chave: participação social, inovação agrícola, ambiente.

\begin{abstract}
This paper proposes to base social participation in productive processes based on local agricultural innovation projects. Themes that affect the social development of rural communities. It is intended to systematize results obtained in the project during its first three stages of experiences, from the sociological perspective of participatory practices associated with agricultural innovation projects. The link established between these categories, favors favorable scenarios for the acquisition of knowledge by rural communities. This guarantees the protection of the environment, healthy food, organic farming and social actors aware of their role in these results.
\end{abstract}

Keywords: social participation, agricultural innovation, environment. 


\section{RELACIÓN DE LA PARTICIPACIÓN SOCIAL CON ESCENARIOS PRODUCTIVOS}

No existe agricultura eficiente sin una buena selección de los cultivos según vocación del suelo, como no existe sostenibilidad agrícola sin un adecuado manejo de los recursos naturales asociados a este proceso, y lo cual solo es posible con la acción del hombre, sujeto activo, como centro del proceso productivo.

Esto demuestra que el manejo adecuado en la producción de bienes (alimentos) transita por un encuentro de saberes, es decir, poner en manos de la comunidad los resultados de la ciencia para de conjunto con sus conocimientos ancestrales se pueda construir un saber que conlleve a la sostenibilidad. Este proceso transita por una desconstrucción de conocimientos y hábitos para construir un nuevo pensamiento más acabado sobre la base del anterior con aportes científicos y renovadores.

Pero esta situación no ha sido precisamente el escenario que se expone hoy en las zonas rurales y más específicamente en ecosistemas montañosos donde ha existido un distanciamiento entre las ciencias encargadas tanto de la producción agrícola como la protección del ambiente, y las características sociológicas de las comunidades.

Es este escenario, precisamente, el antecedente para la investigación proponiéndose esclarecer los vínculos existentes en la comunidad, en cuanto a la relación de los componentes social y ambiental para la producción de alimentos como principales aspectos del desarrollo comunitario en regiones agrícolas.

Múltiples esfuerzos se han realizado durante décadas para mejorar dicha situación, pero muchos han resultado infructuosos pues no se ha visto al sujeto como ente transformador de su propia realidad con aptitudes y saberes propios que le propicien un escenario de cambio en su entorno productivo.

Este ha sido precisamente el caso del proyecto PIAL (Programa de Innovación Agropecuaria Local) implementado en Cuba desde el año 2001, abarcando diferentes provincias del país con municipios seleccionados como 
sitios pilotos de trabajo. Dicho proyecto se encuentra en los momentos actuales en su cuarta etapa de ejecución. Propiciando alternativas válidas para ser implementadas en el actual y futuro contexto cubano.

Como se ha explicado con anterioridad aún se encuentra en ejecución, por tanto, los resultados que aquí se exponen son derivados de etapas anteriores centrando la atención en la participación social como categoría sociológica de análisis tratada en el proyecto PIAL en diferentes momentos.

Es precisamente esta categoría sociológica el hilo conductor de posteriores estudios de investigación a partir de la sistematización de experiencias, incluyendo la dimensión ambiental como herramienta metodológica al proyecto PIAL en la provincia Villa Clara, resultado previsto obtener al cierre de su cuarta etapa (2021).

El objetivo de este trabajo consiste en fundamentar la participación social en procesos productivos a partir del Proyecto PIAL. Tomando como fundamento práctico para ello, resultados de las tres etapas primeras del proyecto, asentados en el informe ejecutivo del proyecto PIAL y propuesta de la cuarta etapa de proyecto, documento elaborado por el grupo coordinador del mismo con la colaboración internacional de COSUDE (Agencia Suiza de Cooperación al Desarrollo).La participación social constituye el modo en que funcionalmente resulta posible la acción colectiva del grupo como sujeto de la actividad. Es en este marco que la actividad alcanza toda la riqueza de sus manifestaciones en el planteamiento y debate de opciones, la toma de decisión, la ejecución y el control de estas, etc.

Concebir la participación como involucramiento activo de los actores sociales en tanto sujetos de la acción social constituye un elemento nuclear que expresa el vínculo de simetría presente en diversas gradaciones dentro de las relaciones grupales en la medida en que el involucramiento activo en la actividad se produce como sujeto de la misma.

Su negación reduce dichos actores a objeto o medio de la actividad como manifestación de un vínculo de asimetría presente en las relaciones en que transcurre el proceso inclusivo. De ahí que no sea suficiente considerar el involucramiento en la conceptualización de participación sin agregar la 
condición (sujeto, medio u objeto) en que este se produce en términos comunitarios (Alonso, 2016).

Respecto al papel de la participación de los individuos en las comunidades Alonso (2016) cita al autor Wrigth Mills, (1969), quien plantea: la práctica social debe ser comprendida como actividad humana que al transformar la sociedad y la naturaleza transforma también al sujeto que la ejerce. Como proceso -y concepto- manifiesta y contiene la unidad de la teoría y la práctica tanto en el ámbito de la producción de bienes materiales como en el de la producción intelectual.

A decir de la importancia que retoma en estas investigaciones el estudio de la participación como categoría de análisis para desarrollas las mismas varios autores han expresado sus criterios desde sus posiciones en diferentes estudios, tales como: Alonso (2009), Alonso, Riera y Rivera (2013) y Alvaro (2015) haciendo un análisis propio del autor con un posicionamiento más profundo desde la interpretación de los clásicos de la sociología (Marx, Tönnies y Weber), y Alonso y Jara (2016).

De igual manera es importante el análisis de la categoría sostenibilidad ambiental para el desarrollo de esta investigación sobre la cual existen algunos referentes desde la sociología ambiental como son estudios realizados por: Parayotou (1994), Delgado (1999) y Leff (2002).

El sistema social cubano propicia la participación de todos los individuo en los diferentes ámbitos y la búsqueda de alternativas de solución a diferentes problemáticas que inciden en el desarrollo y en el mejoramiento de la calidad de vida de las personas, (Aguilera, 2017).

Tal es el caso que se analiza, encaminada a la mejora ambiental en zonas rurales desde la relación armoniosa sociedad-ambiente-producción, situación que se ha visto afectada por mucho tiempo dada la presión de planes de producción y desconociendo en materia de medio ambiente y protección de los recursos naturales de forma sostenible.

Dicho proyecto persigue mejorar la calidad de vida de la población en los municipios involucrados en él a partir del fortalecimiento del sistema de innovación agropecuario en el Desarrollo Local. En el caso específico de Villa 
Clara, el mismo se gestiona desde la Universidad Central "Marta Abreu"4 de Las Villas; encontrándose todo un tejido armónico que propicia la articulación y ejecución de dicho proyecto en estrecho vínculo con otras instituciones.

Poniendo énfasis en el sector agrario campesino, teniendo a la participación social como categoría sociológica que propicia este vínculo entre Centros de ciencia y campesinos. Dicho proyecto en sus diferentes etapas ha ido superando obstáculos través de la innovación y la participación social en el sector agrario, propiciando escenarios productivos donde la agroecología desde diferentes aristas también propicia aportes a la producción de alimentos, capacitación de productores y fortalecimiento institucional (Figura 1).

\footnotetext{
${ }^{4}$ Sitio de la Universidad Central Marta Abreu de las Villas: $<$ https://www.uclv.edu.cu/ $>$, acesso 02/02/2020.
} 


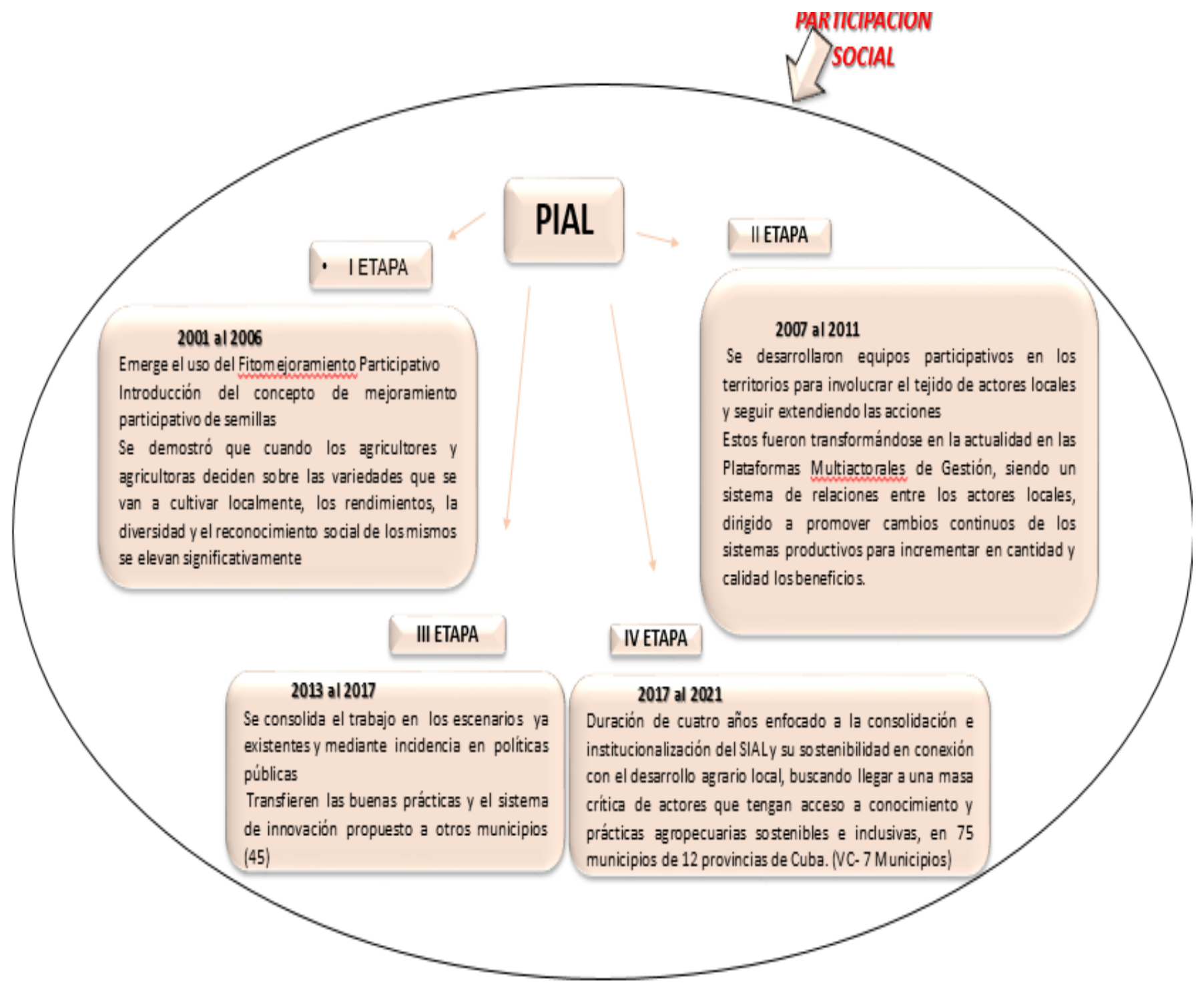

Figura 1: Vínculo de la participación social en el desarrollo del proyecto PIAL en sus cuatro etapas de ejecución.

Es importante también referir que se entiende por procesos productivos una serie de actividades destinadas a la elaboración de un bien o servicio (monografías.com, 2019) los mismos son vistos en cuatro dimensiones:

$\checkmark$ Agroecológica: disponibilidad de recursos naturales.

$\checkmark$ Tecnológica: prácticas culturales, innovaciones tecnológicas.

$\checkmark$ Sociopolítica: actores sociales intervinientes. Regulaciones y normativas estatales. 
$\checkmark$ Comercial: actores y procesos de distribución. Demandas de los mercados (tanto internos como externos).

Durante la primera etapa de ejecución del proyecto se observa que emerge el uso por primera vez del Fitomejoramiento Participativo, dicho periodo abarca los años comprendidos del 2001 al 2006, en el mismo resalta la introducción del concepto de Mejoramiento Participativo de Semillas.

También se demostró que cuando los agricultores y agricultoras deciden sobre las variedades que se van a cultivar en el ámbito local, los rendimientos, la diversidad y el reconocimiento social de los mismos se elevan significativamente, demostrando de esta manera la significación que alcanza el ser considerado parte del proceso de obtención final del producto desde el inicio, lo cual es conocido sociológicamente como participación social del individuo en el proceso. Las figuras 2 y 3 demuestran fehacientemente lo antes expuesto.

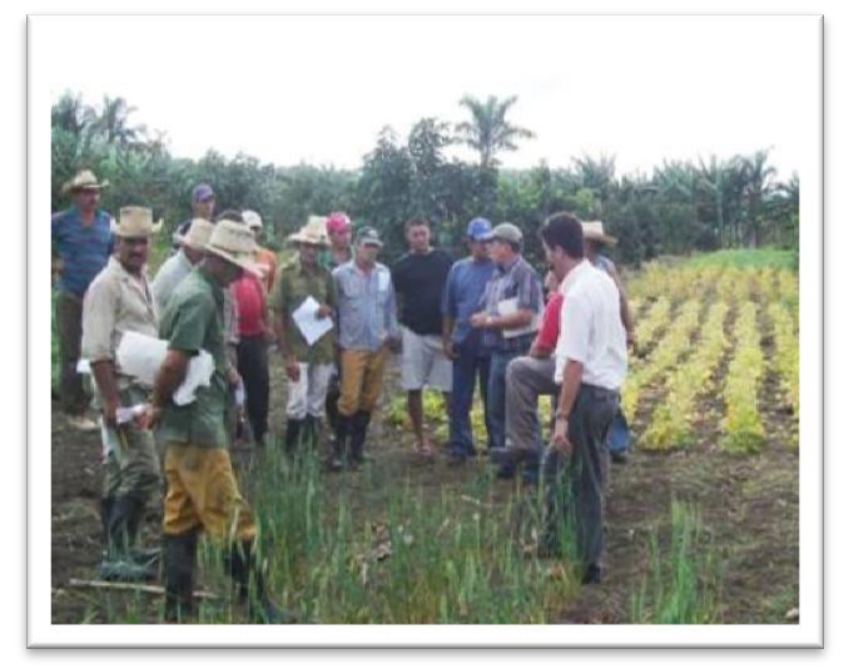

Figura 2: Capacitación en áreas productivas en mejoramiento de semillas a productores de manera participativa. 


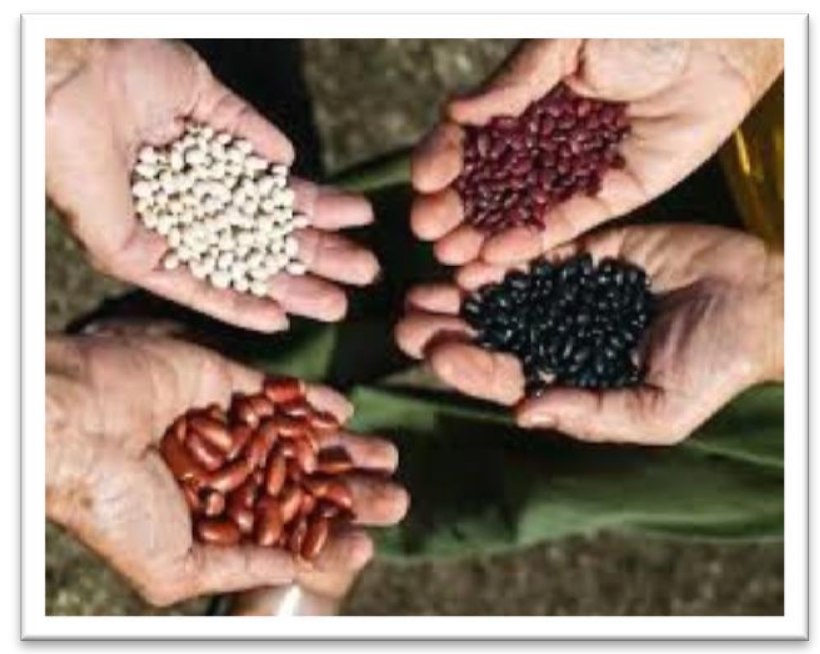

Figura 3: Resultados productivos del mejoramiento de semillas en variedades de granos.

Para la segunda etapa, la cual se enmarca en los años comprendidos desde el 2007 al 2011, el cual implicó diferentes territorios, en los mismos fueron conformados y desarrollaron equipos participativos para involucrar el tejido de actores locales y seguir extendiendo las acciones de trabajo.

Estas acciones, fueron transformándose en el tiempo a lo que en la actualidad es conocido como Plataformas Multiactorales de Gestión (PMG); convirtiéndose en un sistema de relaciones entre los actores locales, dirigido fundamentalmente a promover y desarrollar de manera sostenible, cambios continuos de los sistemas productivos de forma tal que propicie el incremento en cantidad y calidad de los beneficios obtenidos (figuras 4 y 5).

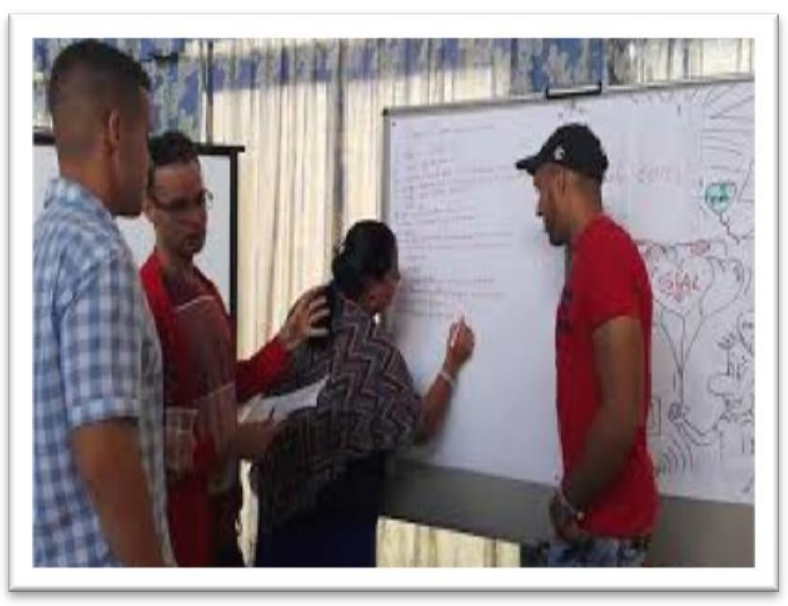

Figura 4: Grupo de formadores y promotores de instituciones educativas en charlas y talleres a productores. 


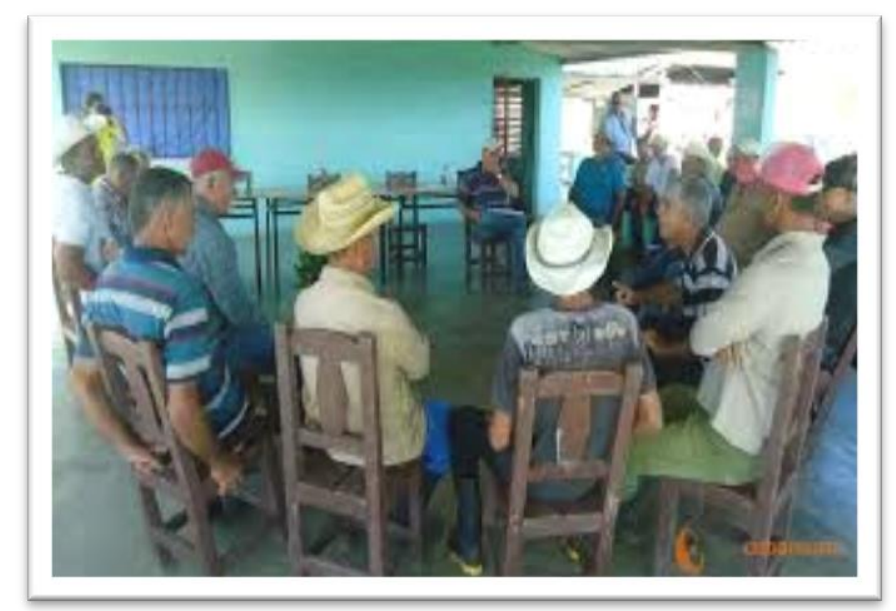

Figura 5: Encuentro practico de promotores agroecológicos con productores implicados en el proyecto.

Por su parte, durante la tercera fase de ejecución, desarrollada la misma en el periodo comprendido del año 2013 al año 2017, se consolida el trabajo en los escenarios ya existentes, dígase sitios de intervención identificados previamente, y mediante incidencia en políticas públicas.

De esa manera se transfieren las buenas prácticas productivas con técnicas agroecológicas y el sistema de innovación propuesto a otros municipios implicados, haciendo así replica de resultados para extenderlos a otros sitios necesitados de estos saberes e incorporarlos e sus procesos productivos.

Se ha logrado que se establezcan en 45 municipios del país, Plataformas de Interacción, Intercambio y Aprendizaje en la acción con la participación de actores claves y los Grupos de Innovación Agropecuarios Locales, consolidados como espacios de Participación Local para el fomento de la Innovación Agropecuaria Local en base a procesos y dinámicas participativas.

El Sistema de Innovación Local que se promueve, articula una estructura básica de la organización social de la innovación, ciclos de gestión de la innovación y de capacidades creadas para aplicar los principios y buenas prácticas en respuesta a las demandas locales de desarrollo. Lo cual ha sido posible con la experticia y capacidad de replicar conocimiento de un grupo 
coordinador a los diferentes escenarios de trabajo identificados en el territorio, a partir de identificar potencialidades y deficiencias a resolver (figura 6).

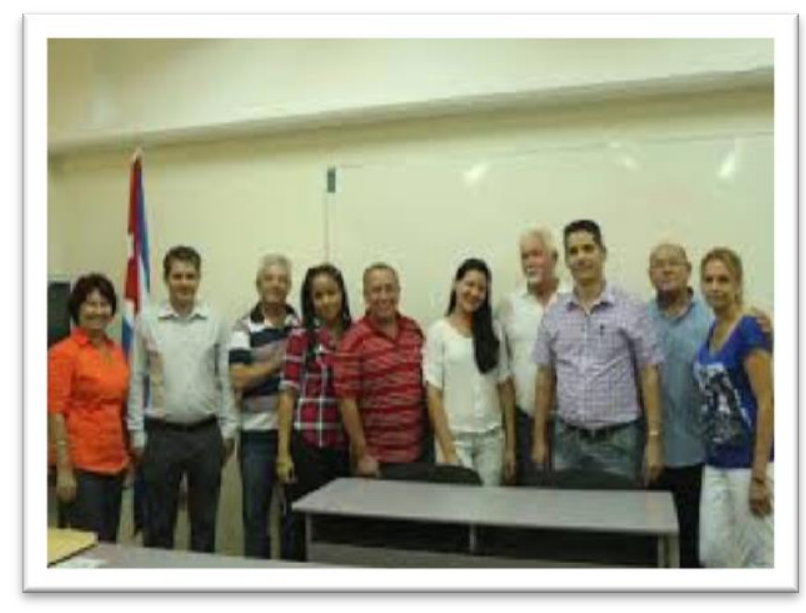

Figura 6: Grupo coordinador implicado en el PIAL en Villa Clara, proveniente de la Universidad Central "Marta Abreu" de Las Villas.

Cuba es un país donde una parte importante de los alimentos que consume su población se importan, el sector cooperativo y privado explotan el $70 \%$ de la tierra produciendo un porcentaje elevado de los alimentos. Actualmente este sector representa a más de 500,000 pequeños agricultores, por tanto, la agricultura está necesitada de un sistema de innovación que llegue de forma efectiva a cubrir sus demandas de tecnologías y conocimiento de todas las formas productivas.

PIAL en todos estos años ha trabajado la innovación agropecuaria local enfocada a la demanda de las cadenas agroalimentarias rurales, inicialmente con el acceso a las semillas, tecnologías y actualmente enfocada a proporcionar un Sistema de Innovación Agropecuaria Local (SIAL) sobre la base de sus experiencias y buenas prácticas.

En la actualidad en Cuba, se trabaja en contribuir a mejorar la gestión cooperativa, fortalecer las capacidades locales, ajustar las cadenas productivas y apoyar iniciativas económicas que le aportan valor añadido a la agricultura. 
El "Proyecto para fortalecer un Sistema de Innovación Agropecuaria en el Desarrollo Local (PIAL)" en su cuarta etapa tiene una duración de 4 años (2017-2021) y está dirigido a la consolidación e institucionalización del SIAL y su sostenibilidad en conexión con el desarrollo agrario local, buscando llegar a una masa crítica de actores que tengan acceso a conocimiento y prácticas agropecuarias sostenibles e inclusivas, en 75 municipios de 12 provincias de Cuba.

Si no se generan mayores niveles de participación de los productores en la búsqueda de soluciones a los problemas existentes todos los esfuerzos anteriores serán minimizados. Potenciar las capacidades creativas y de experimentación, así como posibilitar espacios de articulación, intercambio y construcción colectiva en los escenarios productivos, posibilita aumentar la cifra de participación de los cientos de productores de un municipio, marcando la diferencia para lograr resultados superiores que se reflejen en el acceso de la población a alimentos nutritivos, variados y a precios módicos.

Como resultados propuestos para esta nueva etapa o proyecto se cuentan con metodologías de trabajo que proveen de conocimiento y opciones a los agricultores y actores locales de las cadenas agroproductivas, como son:

$>$ Diplomado SIAL

$>$ Los ciclos de aprendizaje en la acción

$>$ Los intercambios de experiencias

$>$ Ferias de agrodiversidad

$>$ Diagnóstico local de semillas

$>$ Festivales

> Las capacitaciones temáticas contextualizadas con un enfoque muldisciplinario, inclusivo, horizontal y participativo.

Este proyecto a nivel nacional será gestionado por el Instituto Nacional de Ciencias Agrícolas articulando sus acciones con la red GUCID del MES y con la dirección de innovación del MINAG y la dirección de ciencia y técnica del CITMA, representados todos en la Junta Nacional del proyecto. A nivel 
provincial se trabajaremos con 11 instituciones contrapartes involucradas en la gestión estratégica y económica del proyecto y que atenderán directamente a las instituciones municipales en la adopción e implementación del SIAL.

El enfoque de cadenas agroproductivas donde se analice la comercialización, el procesamiento y los servicios de forma conectada e integral; la conservación de los recursos naturales fundamentales (hídricos, agrodiversidad, suelos, bosques); el cambio climático, el acceso a nuevas tecnologías, la disminución de la fuerza de trabajo en la agricultura por el envejecimiento poblacional, la migración de los jóvenes rurales hacia zonas más urbanas, la insuficiente participación y liderazgo de las mujeres en los escenarios agro-productivos, son otros elementos de gran importancia tratados en el proyecto desde su concesión.

La participación activa de los agricultores, técnicos y otros actores en la búsqueda de las soluciones a problemáticas locales de las cadenas agroalimentarias son motivadas y promovidas por las nuevas formas de gestión que aprovechen las potencialidades locales constituyendo a su vez un desafío para ellos.

En la etapa anterior de PIAL se han consolidado estos espacios de participación horizontales para la innovación agropecuaria en 45 municipios, con la conformación de 45 Plataformas Multiactorales de Gestión (PMG), conectadas con 291 Grupos de Innovación Agropecuaria Local (GIAL). Las plataformas son reconocidas y aprovechadas por los gobiernos municipales para gestionar las demandas de desarrollo en el ámbito agropecuario municipal. En estos espacios horizontales las instituciones y los agricultores se reúnen con el gobierno para resolver problemas concretos de las cadenas agroalimentarias.

El enfoque incluye la aplicación de buenas prácticas de participación, inclusión y aprendizaje interactivo aprovechando la red de relaciones que se ha ido formando a lo largo de estos años. Los resultados logrados son significativos: $181^{\prime} 000$ personas (36\% mujeres) se han beneficiado de manera directa por las acciones del proyecto, aumentando la producción de alimentos en un $30 \%$ y sus ingresos proviniendo también de actividades de 
transformación en 50\%, gracias a mecanismos de gestión de conocimientos (plataformas multiactores, ferias, festivales, intercambios, talleres de capacitación etc.).

Al identificar y crear oportunidades para las mujeres, a su vez concientizar en el tema equidad de género y ofrecer capacitación a los actores del proyecto, el programa seguirá incorporando la perspectiva de género. Se promoverá la participación real de las mujeres en todas las actividades productivas y en particular las actividades generadoras de ingresos (miniindustrias, agroturismo, comercialización), contando con estrategias diferenciadas entre municipios con plataformas consolidadas donde se buscará una mayor participación de mujeres en los espacios (PGM, GIAL) y nuevos municipios.

La Red de Doctorandos es un tejido conformado por aspirantes al grado científico de doctor en ciencias, cuyas investigaciones visibilizarán los resultados teóricos, metodológicos y prácticos de la Innovación Agropecuaria Local en interés de vincular la gestión del conocimiento y la innovación, con el desarrollo agropecuario local, el mismo fue creado de manera participativa en el mes de junio del 2016.

Las personas que conforman la Red provienen de distintas universidades, de los Centros Universitarios municipales (CUM), centros de investigaciones, de otras empresas y organismos de las provincias donde actúa el PIAL. Entre sus funciones principales se encuentran:

$\checkmark$ Viabilizar los procesos investigativos en torno a la Investigación Agropecuaria Local

$\checkmark$ Apoyar a investigación y promoción de procesos participativos de desarrollo

$\checkmark$ Articulación de actores que acompañan e investigan procesos de innovación; compartir información, conocimientos, experiencias en torno a la investigación en IAL

$\checkmark$ Facilitar el acceso a la información para doctorandos; visibilizar la red y sus resultados en la comunidad científica nacional e internacional. 
Pero no solo los resultados obtenidos y propuestos están en función de fortalecer la dimensión sociológica desde la categoría de análisis participación social, sino que de forma sinérgica desde esta posición sociológica se promueve resolver problemas ambientales y productivos como parte de la multicondicionalidad de la ciencia. En tal sentido el proyecto a favor de la protección del ambiente, los recursos naturales y mitigar efectos del cambio climático, seguirá promoviendo bio-insumos, buenas prácticas agroecológicas, producción limpia y energías renovables (biogás), de esta manera se garantiza la producción de alimentos.

Un elemento importante de sostenibilidad será el fortalecimiento de las plataformas multiactorales de gestión, se priorizará consolidar las plataformas existentes (45), en particular asegurar que los GIAL (donde están los agricultores) participen activamente y sus demandas de innovación sean tomadas en cuenta por los demás actores: las autoridades municipales, las ONGs especializadas, las universidades, delegaciones de los ministerios en el territorio, proyectos internacionales y locales e incluso los bancos.

Las plataformas deben ser útiles para los distintos actores locales y deben permitir el contacto entre productores, técnicos, especialistas y decisores para llegar a soluciones para el buen funcionamiento de las cadenas agroalimentarias a nivel local.

En las plataformas nuevas (30), se aprovechará la experiencia de las plataformas más avanzadas y sistematizadas en la fase actual para llegar más rápidamente a la conformación del espacio de articulación horizontal, para así dotarlas de mecanismos de funcionamiento comprobado en los municipios cercanos, sin dejar de tener su propia identidad de funcionamiento.

Un nuevo elemento de intervención será la activación de la Red del Sistema de Innovación Agropecuaria Local $^{5}$, la cual, fomentará la

\footnotetext{
5 Ver detalhes: IX Conferencia Científica Internacional Desarrollo Agropecuario y Sostenibilidad AGROCENTRO 2019, <https://convencion.uclv.cu/es ES/event/ix-conferenciacientifica-internacional-desarrollo-agropecuario-y-sostenibilidad-agrocentro-2019-vii-simposiode-procesos-de-innovacion-rural-y-iv-taller-con-productores-innovadores-2019-06-24-2019-0629-24/track>; ou "Crecen en Cuba las redes municipales de innovación agropecuária", in: $<$ http://www.rebelion.org/noticia.php?id=246889 >, acesso 02/02/2020 [Inserção do editor].
} 
sostenibilidad y un accionar sistemático en el impulso del desarrollo del SIAL. Se priorizó la participación de los formadores preparados en el diplomado nacional ejecutado durante los meses de enero a julio del año 2017 y posteriormente un segundo grupo de formación cuyo momento de implementación transcurrió en el año 2018.

Se sumarán todos los formadores preparados durante los años 2018 y 2019 vinculados a las provincias participantes, insertando de igual manera a los integrantes de los equipos municipales, provinciales y nacionales del proyecto, así también serán incorporados en este entramado social a los docentes de los Centros Universitarios Municipales (CUM) para que se sumen también a red.

Entre los trabajos que se han venido realizando en el año 2019 incluye la experiencia participativa de estudiantes de la Facultad de Ciencias Agropecuarias a modo de convivencia durante una semana con campesinos y acompañados de profesores de dicha institución docente en áreas de intervención del proyecto en la zona del macizo montañoso Guamuhaya, territorio villaclareño.

Esta experiencia culmino en un trabajo de participación donde se exponen criterios vividos de ambas grupos sociales en esa semana, resultando algo muy positivo en el accionar de intercambio entre el conocimiento teórico y la empírea, implicando además a futuras generaciones de productores pues también participaron estudiantes de la enseñanza primaria que conforman el Círculo de Interés de la Comunidad Rural La Herradura (figuras 7, 8, 9,10). 


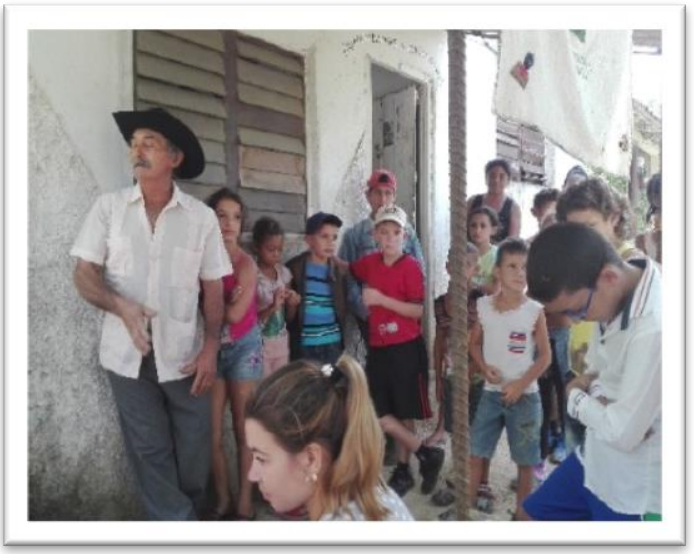

Figura 7: Productor insigne en la Comunidad Rural La Herradura acompañado de pioneros integrantes del Círculo de Interés Ambiental.

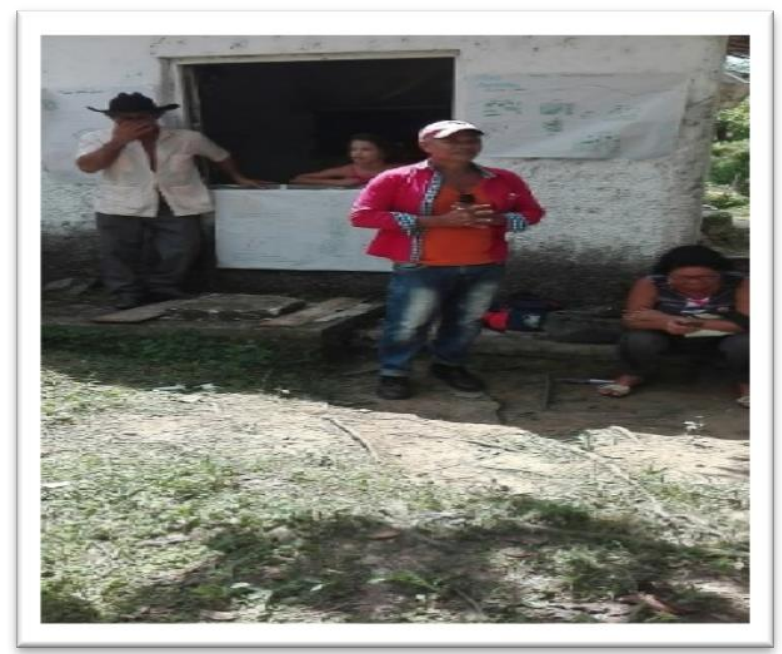

Figura 8: Productor exponiendo sus experiencias y resultados de la convivencia desde su mirada como poblador rural y productor. 


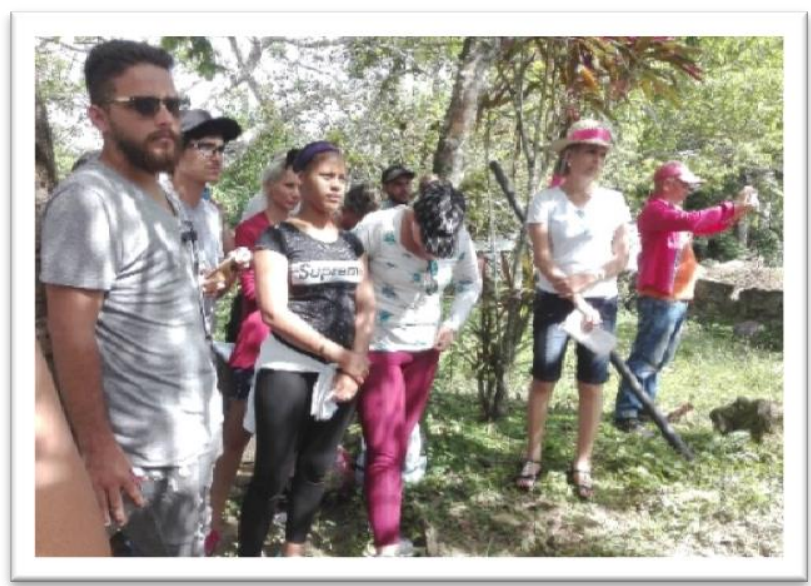

Figura 9: Experiencias expuestas por los estudiantes de la Universidad durante la semana de convivencia en Comunidad Rural.

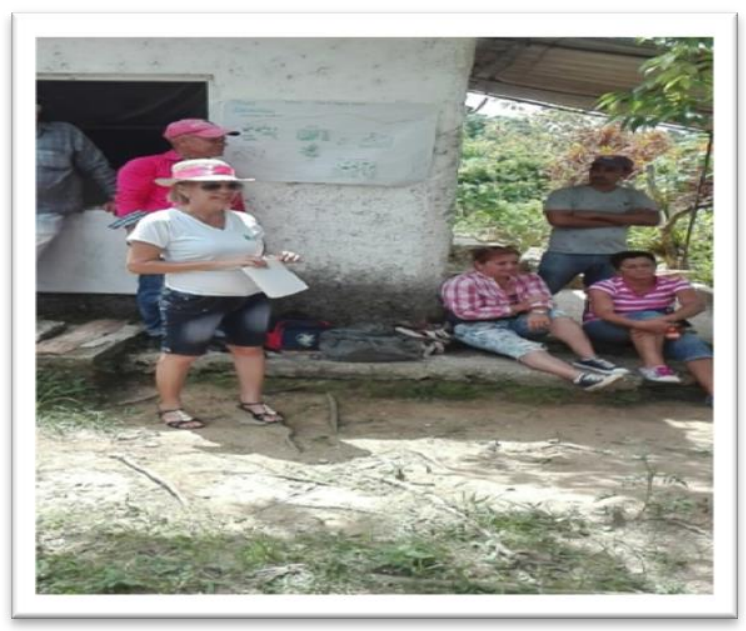

Figura 10: Docente encargada de dirigir el proceso de convivencia con los estudiantes y campesinos en Comunidad Rural. 


\section{CONCLUSIONES}

1. El vínculo que se establece entre participación social y proyectos de innovación agropecuaria en zonas rurales, propicia escenarios favorables para la adquisición de conocimiento por parte de la comunidad rural.

2. La inclusión de la categoría sociológica participación social vinculante en proyectos de innovación agropecuaria local garantiza la protección del ambiente, alimentos sanos, agricultura ecológica y actores sociales conscientes de su papel en estos resultados.

3. La generalización de este proyecto a otras provincias y municipios, favorece el desarrollo sostenible en zonas rurales del país.

4. Los ejercicios de convivencia entre estudiantes, docentes y productores en espacios rurales productivos incentiva la participación y el intercambio de saberes en aras de la producción de alimentos sostenibles. 


\section{REFERENCIAS}

Aguilera L. A y Pérez H. Y, Pérez C. A, G., Cabrera T. L. (2017). La participación del grupo gestor en el proyecto "Ruta Cultura del Undoso", del municipio Sagua la Grande.

Alonso F.J. (2009). La comunidad y lo comunitario en su devenir histórico. Publicado en La responsabilidad individual y organizacional desde un enfoque comunitario. Santa Clara. Editorial Feijoo, 2009. ISBN: 978-959-250-448-6.

Alonso F,J, Riera V.C, Rivero P.R. (2013). Fundamentos conceptuales y metodológicos del autodesarrollo comunitario como alternativa emancipadora. Publicado en: Lo comunitario en la transformación emancipatoria de la sociedad. Editorial Feijoo. Santa Clara. ISBN: 978-959-250-861-3.

Alonso F, J. y Fernández B, R. (2016). La dimensión cultural de la práctica social. Publicado en Islas número 181, año 58, pp. 20-32, enero-marzo.

Alonso F. J, Jara S.D. (2016). Participación diferenciada en proyectos de desarrollo. Ponencia presentada en el Simposio Internacional CIPS-2016 en el marco de la Convención Internacional sobre Ciencia, Tecnología e Innovación. La Habana: Ministerio de Ciencia, Tecnología y Medio Ambiente de la República de Cuba.

Alvaro D. (2015). El problema de la comunidad. Marx, Tönnies y Weber. Ediciones Prometeo libros. Argentina.

Delgado D.C. (1999). Cuba Verde. En busca de un modelo para la sustentabilidad en el siglo XXI. Elecciones, compilaciones y ediciones científicas. Editorial José Martí.

Leff E. (2002). Saber ambiental. Sustentabilidad, racionalidad, complejidad, poder. Siglo XXI editores Argentina S.A.

Parayotou T. (1994). Ecología, Medio Ambiente y Desarrollo. Debate crecimiento vs conservación. Ediciones Gernika S.A. ISBN: 1-55815-244-x.

https://www.monografias.com/docs/Los-procesos-productivos-agropecuarios-yagroindustriales-PKW5VGFJ8UNZ. 2019.

Propuesta de proyecto Internacional. Proyecto para fortalecer un Sistema de Innovación Agropecuaria en el Desarrollo Local (PIAL). 2017-2021. Colectivo de autores. CUBA-COSUDE. 